\title{
Liquid chromatography - high resolution mass spectrometry screening of Astragalus hamosus and Astragalus corniculatus
}

\author{
Aleksandar Shkondrov ${ }^{1}$, Ilina Krasteva ${ }^{1}$ \\ 1 Department of Pharmacognosy, Faculty of Pharmacy, Medical University of Sofia, 2 Dunav St., 1000 Sofia, Bulgaria \\ Corresponding author: Ilina Krasteva (krasteva.ilina@abv.bg)
}

Received 10 November 2020 Accepted 1 December 2020 Published 8 January 2021

Citation: Shkondrov A, Krasteva I (2021) Liquid chromatography - high resolution mass spectrometry screening of Astragalus hamosus and Astragalus corniculatus. Pharmacia 68(1): 135-139. https://doi.org/10.3897/pharmacia.68.e60621

\begin{abstract}
Astragalus hamosus and Astragalus corniculatus were examined for the presence of flavoalkaloids, acylated and highly glycosylated flavonoids. Non-purified extracts of the overground parts of the species were subjected to ultra-high performance liquid chromatography - high resolution electrospray ionisation mass spectrometry (UHPLC-HRESIMS) analysis and the results were compared to authentic reference substances. A flavoalkaloid of kaempferol was newly identified in an extract of A. hamosus. In addition, three compounds - quercetin and kaempferol flavonoids, acylated with hydroxymethylglutaric acid and alcesefoliside, were found in extracts of $A$. hamosus and A. corniculatus for the first time.
\end{abstract}

\section{Keywords}

Astragalus, qualitative analysis, flavoalkaloids, flavonoids, UHPLC-MS

\section{Introduction}

Astragalus hamosus L. (Fabaceae) is a prostrate or ascending annual or biennial herbaceous plant, distributed in Southern Europe, the Mediterranean, Caucasus, Central and Southwest Asia. In Bulgaria it is spread on the Black Sea coast, North-eastern Bulgaria, in Stara Planina Mt., Thracian lowland, the Rhodope Mt., Tundzha hilly plain (Asyov et al. 2012). The flavonols rhamnocitrin-3-O-glucoside (Toaima 2002), rhamnocitrin-4'- $\beta$-D-galactopyranoside, hyperoside, isoquercitrin, astragalin, rhamnocitrin-3-O-neohesperidoside (Krasteva et al. 2007; Krasteva 2013) were isolated from the aboveground parts. In callus and suspension cultures of the species rutin, astragalin and isoquercitrin (Ionkova and Alfermann 1990; Ionkova 1995) and later in introduced samples of the plant hyperoside, astragalin and isoquercitrin (Krasteva et al. 2007) were identified. When rhamnocitrin-4'- $\beta$-D-galactopyranoside was administered with Cisplatin or Gentamicin, a protection of human kidney cells HEK-293T against the cytotoxic effects of nephrotoxic drugs was observed (Krasteva et al. 2008). The antiproliferative activity of a saponin mixture obtained from the herbs of $A$. hamosus, comprised of two saponins, was examined on human malignant cell lines (HL-60, HL-60/Dox, SKW-3, RPMI-8226, U-266 and OPM-2) using the MTT test (Krasteva et al. 2008). It has been found that the saponin mixture caused concentration-dependent suppression of the proliferative activity of malignantly transformed cells, confirmed by ELISA test to assess apoptosis-specific DNA fragmentation (Dineva et al. 2010). Two oleanane-type saponins - peregrinozide I and azukisaponin V from $A$. hamosus did not show cytotoxic activity against various human tumour cells, but a 
dose-dependent modulation of lymphocyte proliferation was observed (Verotta et al. 2002). Volatile compounds from the species were tested for cytotoxic activity in vitro. A well-defined concentration-dependent antiproliferative activity has been established on REH cells (acute human lymphoid leukaemia) (Momekov et al. 2007). Rhamnocitrin- $4^{\prime}-\beta$-D-galactopyranoside exhibited hepatoprotective activity against $\mathrm{N}$-diethylnitrosamine (DENA)-induced liver cancer in Wistar rats (Saleem et al. 2013). A hydro alcoholic extract, as well as hexane and ethyl acetate fractions obtained from the fruits of the plant exhibited anti-inflammatory and analgesic activity in various animal models (Shojaii et al. 2015).

Astragalus corniculatus Bieb. (Fabaceae) is a perennial herbaceous plant with well-developed roots. In Europe the species is distributed in Romania, Ukraine, and Moldova. It is novel to Bulgarian flora and found in the Danube Plain (Pavlova 1988). From the aerial part of the plant tree pentacyclic triterpene saponins were isolated: 3 -O- $\beta$-[4-oxo- $\beta$-pentopyranosyl- $(1 \rightarrow 2)-\beta$-Dglucopyranosyl]-21 $\alpha$-hydroxy oleanoic acid, 3-O- $\beta$-4oxo-pentopyranosyl-21 $\alpha$-hydroxy oleanolic acid and 3-O- $\beta$-D-xylopyranosyl-3 $\beta, 19 \alpha$-dihydroxy-olean-12ene-28,21 $\beta$-olide (Krasteva et al. 2006; Krasteva et al. 2007). Flavonoids identified are vitexin, orientin, eryodictiol-7-O-glucoside, isorhamnetin-3-O-glucoside, rutin, hyperoside, isoquercitrin, isorhamnetin-3-O-rutinoside, quercetin, kaempferol, isoramnetin, eriodictiol-7-O-rutinoside, quercitrin, floridzin and homoerydictiol (Krasteva 2013). A standardised ethyl acetate extract from $A$. corniculatus was found practically non-toxic $\left(\mathrm{LD}_{50}>5000 \mathrm{mg} / \mathrm{kg}\right)$, showed an antihypoxic effect in a model of chemical hypoxia, and particularly favourably affected circulatory hypoxia (Krasteva et al. 2004). Volatile fractions from the aboveground parts were cytotoxic against a panel of human malignant cells (Krasteva et al. 2008). In series of studies, a purified saponin mixture (PSM) had protective effects against the invasiveness of bone marrow cancer in hamsters. Treatment of animals with implanted tumours with the saponin mixture resulted in an increase in the number, migration, and phagocytic index of peritoneal macrophages and blood polymorphonuclear leukocytes and there was an evidence of an immunostimulatory effect (Toshkova et al. 2007; Toshkova et al. 2008). Statistically significant antioxidant and hepatoprotective activity has been established in vitro for the same PSM on enzyme- and non-enzyme-induced lipid peroxidation in liver microsomes derived from spontaneously hypertensive rats (Simeonova et al. 2010). These effects have been confirmed in other in vivo and in vitro models of hepatic impairment (Vitcheva et al. 2013).

In continuation of our efforts to gain knowledge on the chemical composition of Bulgarian Astragalus species, an ultrahigh performance liquid chromatography - electrospray ionisation mass spectrometry (UHPLC-HRESIMS) screening for the presence of rare flavoalkaloids, acylated and highly glycosylated flavonoids in A. corniculatus and A. hamosus was performed.

\section{Materials and methods}

\section{Plant material and extraction}

The overground parts of A. hamosus were collected in July 2018 from Sofia. The above ground parts of $A$. corniculatus were harvested form Gorna Studena in June 2019. Both plants were in flowering. The identity of the species was confirmed by us (A. S. and I. K.). Voucher specimens were deposited in the Herbarium of the Institute of Biodiversity and Ecosystem Research at the Bulgarian Academy of Sciences: SOM 1398 (A. hamosus) and SOM 1399 (A. corniculatus). The samples were dried at room temperature and then $200 \mathrm{mg}$ of each were extracted twice with $2.5 \mathrm{~mL} 80 \% \mathrm{MeOH}$ on a water bath for $30 \mathrm{~min}$ each (in reflux). The extracts obtained from each sample were filtered, combined in a volumetric flask and the volume adjusted to $10.0 \mathrm{~mL}$ with $80 \% \mathrm{MeOH}$. After filtration through a membrane PVDF syringe filter $(0.22 \mu \mathrm{m})$ an aliquot of $2 \mu \mathrm{L}$ was injected to the UHPLC system.

\section{Ultra high performance liquid chroma- tography-high resolution electrospray ionization mass spectrometry (UH- PLC-HRESIMS)}

A Q Exactive Plus Orbitrap mass spectrometer with a heated electrospray ionisation (HESI) ion source (ThermoFisher Scientific, Bremen, Germany) coupled with a UHPLC system (Dionex UltiMate 3000 RSLC, ThermoFisher Scientific, Bremen, Germany) was used. The full scan MS was set at: resolution 70000 (at $m / z 200$ ), AGC target 3e6, max IT $100 \mathrm{~ms}$, scan range 250 to $1700 \mathrm{~m} / z$. The $\mathrm{MS}^{2}$ conditions were: resolution 17500 (at $\mathrm{m} / z$ 200), AGC target $1 \mathrm{e} 5$, max IT $50 \mathrm{~ms}$, mass range $\mathrm{m} / z 200$ to 2000 , isolation window $2.0 \mathrm{~m} / z$ and (N)CE 20. The ionization device (HESI source) was operating at: +3.5 or $-2.5 \mathrm{kV}$ spray voltage and $320{ }^{\circ} \mathrm{C}$ capillary and probe temperature, 38 arbitrary units (a.u., as set by the Extactive Tune software) of sheath gas and 12 a.u. of auxiliary gas (both Nitrogen); S-Lens RF level 50.0. UHPLC separations were performed on a Kromasil $\mathrm{C}_{18}$ column $(1.9 \mu \mathrm{m}, 2.1 \times 50 \mathrm{~mm}$, Akzo Nobel, Sweden) at $40{ }^{\circ} \mathrm{C}$. The mobile phase was $\mathrm{H}_{2} \mathrm{O}+$ $0.1 \% \mathrm{HCOOH}(\mathrm{A})$ and $\mathrm{MeCN}+0.1 \% \mathrm{HCOOH}(\mathrm{B})$ with a flow rate of $0.3 \mathrm{~mL} / \mathrm{min}$. Elution was as follows: $10 \% \mathrm{~B}$ for $0.5 \mathrm{~min}$, increase to $30 \% \mathrm{~B}$ for $7 \mathrm{~min}$, isocratic with $30 \% \mathrm{~B}$ for $1.5 \mathrm{~min}$, increase to $95 \%$ B for $3.5 \mathrm{~min}$, isocratic with $95 \%$ B for $2 \mathrm{~min}$, return to $10 \%$ B for $0.1 \mathrm{~min}$. Detection of the compounds in plant samples was performed in both the positive and the negative ionisation mode by a set range of $m / z$ of the corresponding protonated or deprotonated molecule with a time filter, adjusted to the retention time of each standard. Identification was supported by $\mathrm{MS}^{2}$ experiments which revealed the aglycone part of the molecule as well as the successive loss of monosaccharides of the sugar moiety. The fragmentation pattern was compared to that of the reference substances. The software Xcalibur, Version 4.2 (Thermo Scientific) was used for data collection and processing. 


\section{Reference substances}

From A. monspesulanus subsp. monspesulanus $N$-(8-methylquercetin-3-O-[ $\alpha$-L-rhamnopyranosyl- $(1 \rightarrow 2)-[\alpha$-L-rhamnopyranosyl- $(1 \rightarrow 6)]-\beta$-D-galactopyranosyl])-3-hydroxypiperidine-2-one (1), $N$-(8-methylkaempferol-3-O- $\alpha$-L-rhamnopyranosyl- $(1 \rightarrow 2)-[\alpha$-L-rhamnopyranosyl-(1 $\rightarrow 6)]-\beta$-D-galactopyranosyl])-3-hydroxypiperidine-2-one(2), quercetin-3-O- $\alpha$-L-rhamnopyranosyl- $(1 \rightarrow 2)$-[6-O-(3-hydroxy-3-methylglutaryl)- $\beta$-D-galactopyranoside] (3), kaempferol-3-O- $\alpha$-L-rhamnopyranosyl- $(1 \rightarrow 2)$-[6-O-(3-hydroxy-3-methylglutaryl)- $\beta$-D-galactopyranoside] quercetin-3-O- $\alpha$-L-rhamnopyranosyl- $(1 \rightarrow 2)$ - $[\alpha$-L-rhamnopyranosyl- $(1 \rightarrow 6)]-\beta$-D-galactopyranoside (alcesefoliside, 5) were obtained by a previously described procedure (purity more than 95\%); from A. glycyphyllos kaempferol-3-O-[2-O- $\beta$-D-galactopyranosyl-6-O- $\alpha$-L-rhamnopyranosyl]- $\beta$-D-glucopyranoside (camelliaside A, 6) (purity 98\%) was isolated as reported before. The compounds were identified by extensive MS and NMR analyses (Krasteva et al. 2015; Shkondrov et al. 2020). Rutin (7) was purchased form Sigma Aldrich (Germany) and used to confirm the accuracy of the analysis. Standard solutions of each reference substance were prepared in $\mathrm{MeOH}(1000 \mathrm{ng} / \mathrm{mL})$. Two $\mu \mathrm{L}$ of each solution were injected in the UHPLC-HRESIMS system three times to obtain the mean retention time.

\section{Results and discussion}

Non-purified extracts of the aerial parts of both species were investigated by LC-MS for the presence of flavoalkaloids, acylated and highly glycosylated flavonoids, using comparison with selected reference substances (both the retention time and the fragmentation pattern). The results are presented in Table 1 .
Table 1. Compounds identified in the samples.

\begin{tabular}{|c|c|c|}
\hline Compound $^{*}$ & A. hamosus & A. cornicilatus \\
\hline 1 & not found & not found \\
\hline 2 & present & not found \\
\hline 3 & present & present \\
\hline 4 & present & present \\
\hline 5 & present & present \\
\hline 6 & not found & not found \\
\hline 7 & present & not found \\
\hline
\end{tabular}

*see Reference substances.

\section{Compounds in A. hamosus}

Six compounds were identified in the extract (Fig. 1) for the first time. The quercetin flavoalkaloid (1) was not present in the sample - neither a peak with $t_{R}$ corresponding to the reference $(2.47 \mathrm{~min}$ ) was found, nor a protonated or a deprotonated molecule with the corresponding $\mathrm{m} / \mathrm{z}$ of that of the reference $\left(m / z 884,[\mathrm{M}+\mathrm{H}]^{+}\right.$or $\left.m / z 882,[\mathrm{M}-\mathrm{H}]^{-}\right)$ was identified. The kaempferol-derived flavoalkaloid 2 was found and had a $t_{R}$ of 3.00 min and matching fragmentation patterns in both ionization modes to that of the reference compound. In the negative mode a chlorine adduct $(\mathrm{m} / \mathrm{z}$ 901.2643, $\left.[\mathrm{M}+\mathrm{Cl}]^{-}\right)$and the kaempferol moiety in the $\mathrm{MS}^{2}$ $(\mathrm{m} / z 284.0328)$ were proved. The protonated molecule was in a very low abundance with $m / z$ 868.2852. The hydroxymethyl acylated flavonol 3 was proved in the sample with $t_{R}$ of 5.79 min and its fragmentation coincided with the reference in both ionization modes. In the negative mode an ion, corresponding to the deprotonated molecule [M$\mathrm{H}^{-}(m / z 753.1900)$ and in the $\mathrm{MS}^{2}$ a quercetin fragment $(\mathrm{m} / z$ 301.0336), also a characteristic fragment for flavonols with $m / z 151$, after retro Diels-Adler reaction (RDA) were recorded. In the positive mode, the protonated molecular ion $(m / z 755.2036)$ and in the $\mathrm{MS}^{2}$ again a quercetin fragment $(\mathrm{m} / \mathrm{z}$ 303.0501) were observed. The kaempferol flavonoid acylated with hydroxymethylglutaric acid (4) had a $t_{\mathrm{R}}$

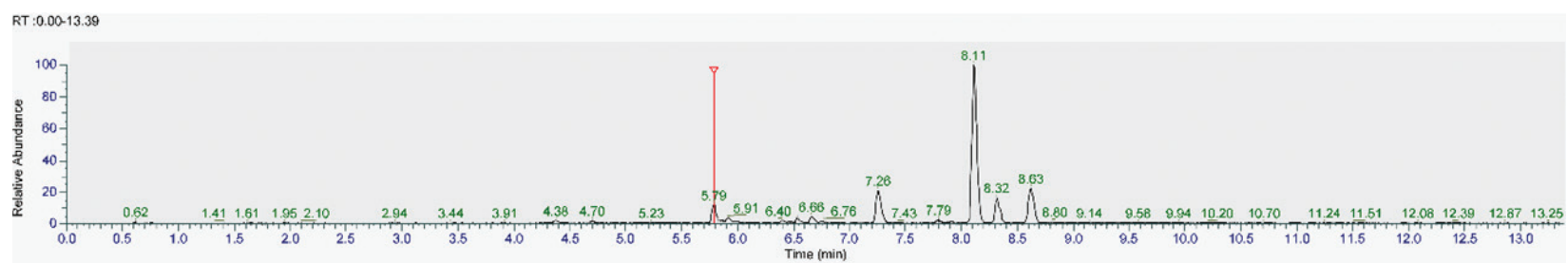
T: FTMS - p $\bar{E}$ SI Full ms $[250.0000-1700.0000]$
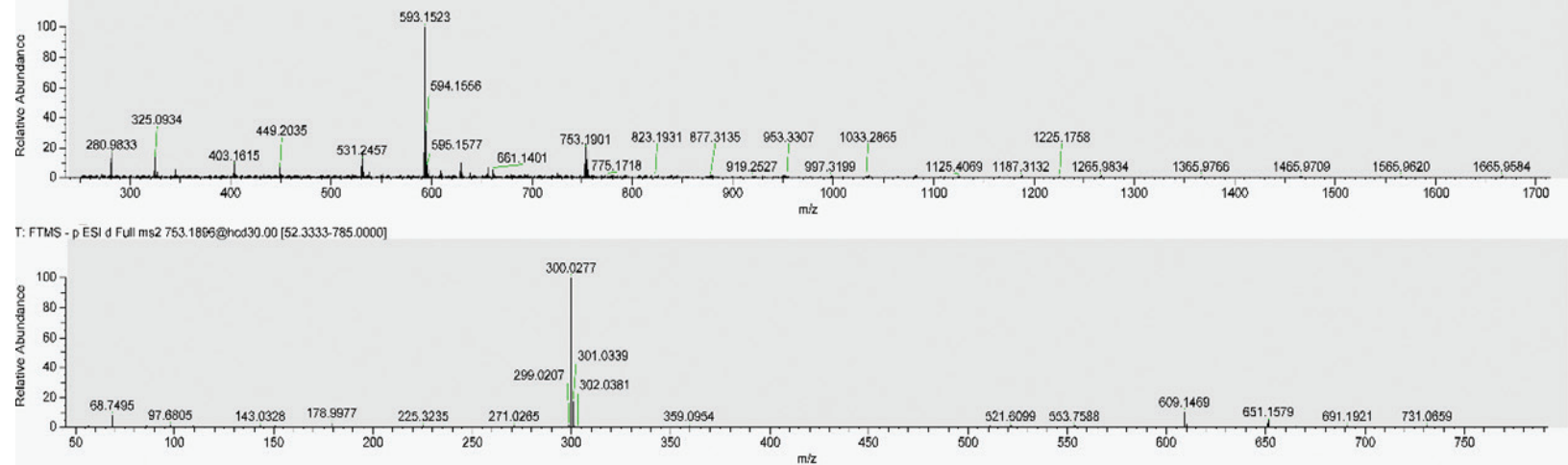

Figure 1. A base peak chromatogram of the extract of A. hamosus with identification of $\left.3(\mathrm{~m} / z 753[\mathrm{M}-\mathrm{H}]]^{-}\right)$and $\mathrm{MS}^{2}$ spectrum displaying quercetin fragment $(\mathrm{m} / \mathrm{z} 301)$. 


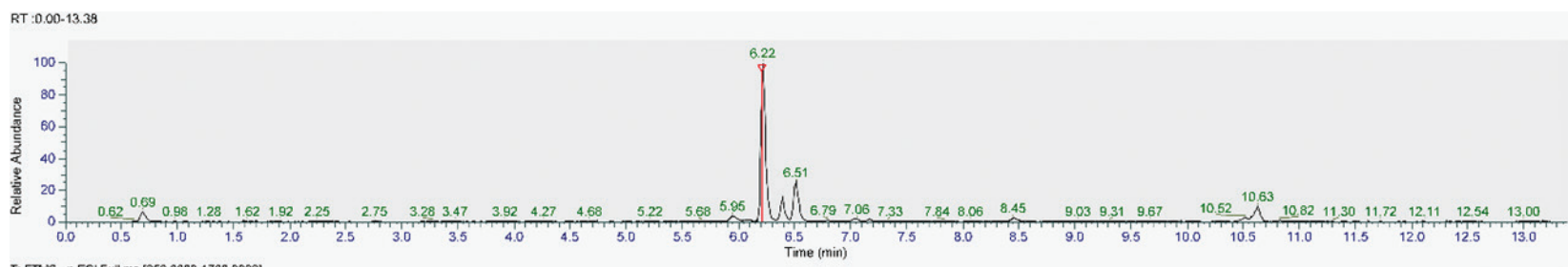
T. FTMS - PESI FUll Ms $25000000-170000000$
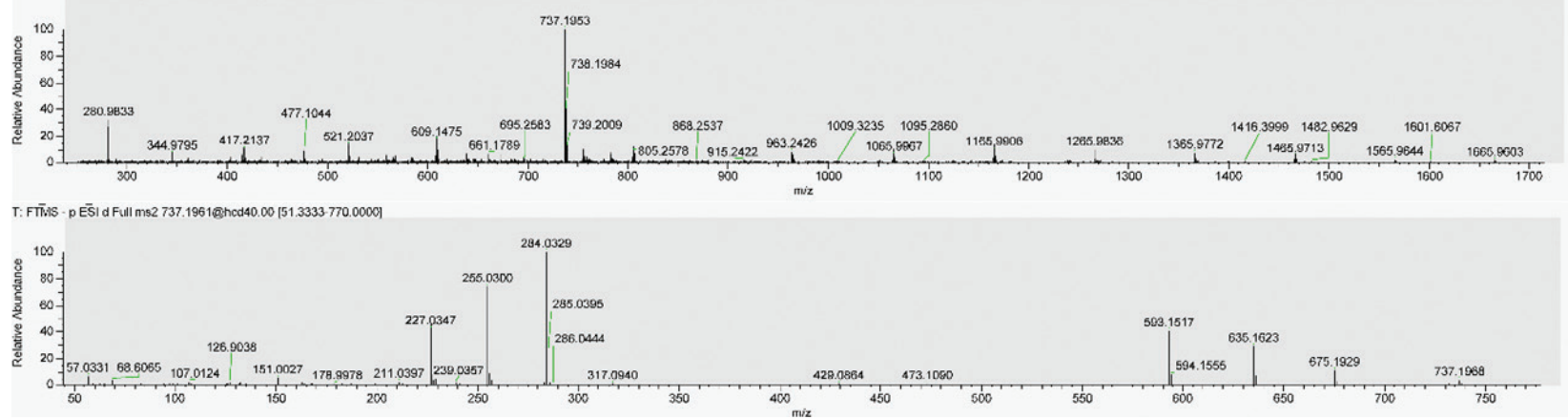

Figure 2. A base peak chromatogram of the extract of $A$. corniculatus with identification of $4\left(\mathrm{~m} / z 737[\mathrm{M}-\mathrm{H}]^{-}\right)$and $\mathrm{MS}^{2}$ spectrum displaying kaempferol fragment $(m / z 284)$.

of $6.21 \mathrm{~min}$, with fragmentation identical to the reference substance in both polarities. In the negative mode an ion [M-H] $]^{-}$with $m / z 737.1953$ and in the $\mathrm{MS}^{2}$ fragment ions of kaempferol $(m / z 284.0329)$ and a flavonol-specific ion $(\mathrm{m} / z 151, \mathrm{RDA})$ were found. In the positive mode the compound was protonated $(\mathrm{m} / z 739.2087)$ with the cleavage of kaempferol moiety $(m / z 287.0551)$ in the $\mathrm{MS}^{2}$. In the sample alcesefoliside (5) was identified as well. The compound had a $t_{R}$ of 4.94 min and the same fragmentation to that of the reference substance. In the negative mode an ion [M$\mathrm{H}^{-}\left(\mathrm{m} / z\right.$ 755.2062) was observed which in the $\mathrm{MS}^{2}$ gave quercetin $(\mathrm{m} / z$ 300.0279) and a flavonol-specific ion after RDA $(m / z 151)$; whilst in the positive mode, a protonated molecule $[\mathrm{M}+\mathrm{H}]^{+}(\mathrm{m} / z$ 757.2195) and quercetin fragment $(\mathrm{m} / z$ 303.0050) were registered. Camelliaside A (6) was not identified in the sample. There was no peak with $t_{\mathrm{R}}$ of 6 (4.99 $\mathrm{min}$ ) and no ions with $\mathrm{m} / z$ corresponding to the protonated or deprotonated molecule $\left(\mathrm{m} / z 757,[\mathrm{M}+\mathrm{H}]^{+}\right.$ or $\left.m / z 755,[\mathrm{M}-\mathrm{H}]^{-}\right)$. In the sample 7 had a retention time of $5.69 \mathrm{~min}$, which in the negative mode gave [M-H],$(\mathrm{m} / \mathrm{z}$ 609.1474), a quercetin fragment $m / z 301.0347$ and the RDA related ion $(m / z 151)$, while in the positive mode it was registered as a protonated molecule $(\mathrm{m} / z$ 611.1601), which fragmented to the same aglycone $(\mathrm{m} / \mathrm{z}$ 303.0496). Rutin was previously found only in suspension cultures of A. hamosus (Ionkova and Alfermann 1990).

\section{References}

Asyov B, Petrova A, Dimitrov D, Vasilev R (2012) Conspectus of the Bulgarian vascular flora. Distribution maps and floristic elements. Bulgarian Biodiversity Foundation, Sofia, 88-91.

Dineva I, Krasteva I, Berger M, Konstantinov S (2010) In vitro antineoplastic activity of some cytoreductive drugs versus new compounds of plant origin. International Journal of Current Chemistry 1(4): 281-290.

\section{Compounds in A. corniculatus}

Only three of the investigated compounds were found in the extract for the first time (fig. 2). The acylated flavonoids (3 and 4) were both present with $t_{R}$ and fragmentation patterns corresponding to the standards (see compounds in A. hamosus). In addition, alcesefoliside $\mathbf{5}$ was discovered in the sample as well. Again, its fragmentation pattern and $t_{R}$ coincided to the reference substance (see above). The compounds 1, 2, 6 and 7 were not found in the sample.

\section{Conclusion}

Using a highly sensitive UHPLC-HRESIMS method a flavoalkaloid of kaempferol, quercetin and kaempferol flavonoids, acylated with hydroxymethylglutaric acid and alcesefoliside, were newly identified in extracts of $A$. hamosus and A. corniculatus. The presence of the quercetin flavoalkaloid and camelliaside A was not proved in any of the samples.

\section{Acknowledgements}

This work was supported by the Council of Medicinal Science at Medical University of Sofia, Contract № D-122/2020.

Ionkova I, Alfermann A (1990) Transformation of Astragalus species by Agrobacterium rhizogenes and their saponin production. Planta Medica 56(06): 634-635. https://doi.org/10.1055/s-2006-961276

Ionkova I (1995) Astragalus species (Milk Vetch): In vitro culture and the production of saponins, astragaline, and other biologically active compounds. In: Bajaj YPS (Ed.) Medicinal and Aromatic 
Plants VIII. Springer, Berlin, 97-138. https://doi.org/10.1007/978-

3-662-08612-4 6

Krasteva I, Momekov G, Zdraveva P, Konstantinov S, Nikolov S (2008) Antiproliferative effects of a flavonoid and saponins from Astragalus hamosus against human tumor cell lines. Pharmacognosy Magazine 4(16): 269-272.

Krasteva I, Bratkov V, Bucar F, Kunert O, Kollroser M, Kondeva-Burdina M, Ionkova I (2015) Flavoalkaloids and flavonoids from Astragalus monspessulanus. Journal of Natural Products 78(11): 2565-2571. https://doi.org/10.1021/acs.jnatprod.5b00502

Krasteva I (2013) Phenolic compounds in four Astragalus species. European Journal of Medicinal Plants 3(4): 616-623. https://doi. org/10.9734/EJMP/2013/4976

Krasteva I, Nikolov S, Kaloga M, Mayer G (2006) Triterpenoid saponins from Astragalus corniculatus. Zeitschrift Fur Naturforschung - Section B Journal of Chemical Sciences 61(9): 1166-1169. https://doi. org/10.1515/znb-2006-0919

Krasteva I, Nikolov S, Kaloga M, Mayer G (2007) A new saponin lactone from Astragalus corniculatus. Natural Product Research 21(10): 941-945. https://doi.org/10.1080/14786410701526008

Krasteva I, Nikolova I, Danchev N, Nikolov S (2004) Phytochemical analysis of ethyl acetate extract from Astragalus corniculatus Bieb. and brain antihypoxic activity. Acta Pharmaceutica 54(2): 151-156. https://hrcak.srce.hr/16826

Krasteva I, Platikanov S, Momekov G, Konstantinov S, Nikolov S (2008) Phytochemical analysis and in vitro cytotoxic activity of volatiles from Astragalus corniculatus. Natural Product Research 22(11): 969-974. https://doi.org/10.1080/14786410701650287

Krasteva I, Platikanov S, Nikolov S, Kaloga M (2007) Flavonoids from Astragalus hamosus. Natural Product Research 21(5): 392-395. https://doi.org/10.1080/14786410701236871

Momekov G, Krasteva I, Platikanov S, Nikolov S, Konstantinov S (2007) Cytotoxic activity of volatiles from four Astragalus species. Comptes Rendus de l'Academie Bulgare Des Sciences 60(9): 1023-1026.

Pavlova D (1988) A taxonomic investigation of Astragalus species represented in Bulgaria. Sofia University St. Kliment Ohridski.

Saleem S, Shaharyar MA, Khusroo MJ, Ahmadm P, Ur Rahman R, Ahmad K, Alam MJ, Al-Harbi NO, Iqbal M, Imam F (2013) Anticancer potential of rhamnocitrin 4 '- $\beta$-D-galactopyranoside against
$\mathrm{N}$-diethylnitrosamine-induced hepatocellular carcinoma in rats. Molecular and Cellular Biochemistry 384(1-2): 147-153. https://doi. org/10.1007/s11010-013-1792-6

Shkondrov A, Krasteva I, Bucar F, Kunert O, Kondeva-Burdina M, Ionkova I (2020) A new tetracyclic saponin from Astragalus glycyphyllos L. and its neuroprotective and hMAO-B inhibiting activity. Natural Product Research 34(4): 511-517. https://doi.org/10.1080/1 4786419.2018.1491040

Shojaii A, Motaghinejad M, Norouzi S, Motevalian M (2015) Evaluation of anti-inflammatory and analgesic activity of the extract and fractions of Astragalus hamosus in animal models. Iranian Journal of Pharmaceutical Research 14(1): 263-269.

Simeonova R, Vitcheva V, Kondeva-Burdina M, Krasteva I, Nikolov N, Mitcheva M (2010) Effect of purified saponin mixture from Astragalus corniculatus on enzyme- and non-enzyme-induced lipid peroxidation in liver microsomes from spontaneously hypertensive rats and normotensive rats. Phytomedicine 17(5): 346-349. https://doi. org/10.1016/j.phymed.2009.08.013

Toaima S (2002) Flavonoidal glycosides of some Astragalus species. Alexandria Journal of Pharmaceutical Sciences 16(2): 135-138.

Toshkova R, Krasteva I, Nikolov S (2008) Immunorestoration and augmentation of mitogen lymphocyte response in Graffi tumor bearing hamsters by purified saponin mixture from Astragalus corniculatus. Phytomedicine 15(10): 876-881. https://doi.org/10.1016/j. phymed.2007.11.026

Toshkova R, Krasteva I, Wesselinova D, Nikolov S (2007) Influence of purified saponin mixture from Astragalus corniculatus Bieb. on phagocytic cells in Graffi-tumor bearing hamsters. Journal of Ethnopharmacology 109(3): 394-399. https://doi.org/10.1016/j. jep.2006.08.006

Verotta L, Guerrini M, El-Sebakhy N, Assad A, Toaima S, Radwan M, Luo Y, Pezzuto J (2002) Cycloartane and oleanane saponins from Egyptian Astragalus spp. as modulators of lymphocyte proliferation. Planta Medica 68(11): 986-994. https://doi. org/10.1055/s-2002-35654

Vitcheva V, Simeonova R, Krasteva I, Nikolov S, Mitcheva M (2013) Protective effects of a purified saponin mixture from Astragalus corniculatus Bieb., in vivo hepatotoxicity models. Phytotherapy Research 27(5): 731-736. https://doi.org/10.1002/ptr.4785 